\title{
An excellent ozone-resistant polymethylvinylsiloxane coating on natural rubber by thiol-ene click chemistry
}

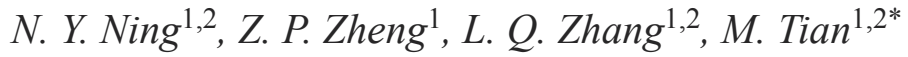 \\ ${ }^{1}$ State Key Laboratory of Organic-Inorganic Composites, Beijing University of Chemical Technology, 100029 Beijing, \\ China \\ ${ }^{2}$ Key Laboratory of Carbon Fiber and Functional Polymers, Ministry of Education, Beijing University of Chemical \\ Technology, 100029 Beijing, China
}

Received 30 September 2014; accepted in revised form 17 December 2014

\begin{abstract}
Natural rubber (NR) as renewable resource is a kind of cheap and versatile elastomer. A disadvantage of NR is that the ozone resistance is not good, which needs to be improved for its wider application. In this study, polymethylvinylsiloxane (PMVS) coating on natural rubber (NR) was realized for the first time by using thiol-ene click reaction under UV irradiation, simultaniously realizing the fast crosslinking of PMVS layer and the covalent crosslinking between PMVS layer and NR layer. As a result, a good interphase adhesion between PMVS coating and NR was obtained. The coating of the crosslinked PMVS layer on NR resulted in an obvious increase in the ozone resistance of NR. Our study provides a new and high efficient strategy to prepare elastomer materials with good ozone resistance.
\end{abstract}

Keywords: coating, the thiol-ene click reaction, interphase adhesion, ozone resistance

\section{Introduction}

Natural rubber (NR) is a cheap and versatile elastomer, and thus has been widely used in industry such as tires. Nowadays, NR as renewable resource has attracted more attention because of the requirement of environmental protection and resource saving. However, the ozone resistance of NR is not good because of its unsaturated chain structure [1]. Numerous works have been conducted to improve the ozone resistance of NR by adding paraffin or antioxidant in NR matrix or by blending NR with other rubbers with good ozone-resistance [2-4]. The disadvantages of these methods are the deterioration of processability and mechanical properties of NR. Hydrogenation of NR is also an effective method to improve the ozone resistance of NR [5]. A disadvantage of this method is that the hydrogenation reaction is complicated. Furthermore, the coating of hydrogenated carboxylated nitrile butadiene rubber (HXNBR) on NR by using the room temperature curing systems was also effective to improve the ozone resistance of NR [6]. But the traditional curing method at room temperature is inefficient.

Silicon rubber has excellent resistance to extremely low and high temperatures $\left(-55\right.$ to $\left.300^{\circ} \mathrm{C}\right)$, excellent elasticity and good ozone resistance. Thus, the coating of silicon rubber on the surface of substrates with good interphase adhesion has been prepared by roomtemperature vulcanizing [7]. However, the adhesion strength between silicon rubber and NR is poor because of the extremely low surface tension of silicone rubber [8]. Thus, the coating of silicon rubber on NR has not been reported to improve the ozone resistance of NR.

The thiol-ene click reaction, which is extremely rapid and copper-free reaction with mild reaction condi-

\footnotetext{
${ }^{*}$ Corresponding author, e-mail: tianm@mail.buct.edu.cn

(C) BME-PT
} 
tions, good selectivity and high yields, has attracted considerable attention in the past few years [9-11]. In previous studies, UV-curing silicon rubber has been prepared by thiol-ene reaction $[12,13]$. In addition, thiol functional groups were successfully introduced on polydimethylsiloxane (PDMS) elastomer, and the thiol-functionalized PDMS was modified with hydrophilic molecules containing enes by using the thiol-ene click reaction [14].

In this study, we used the thiol-ene reaction to simultaniously realize the crosslinking of polymethylvinylsiloxane (PMVS) layer and the covalent crosslinking between PMVS and NR to improve the interphase adhesion between PMVS coating and NR. We prepared PMVS coating on peroxide pre-crosslinked NR sheet by spraying PMVS/trimethylolpropane tris (3-mercaptopropionate) (TMPMP) solution on NR followed by crosslinking reaction via thiol-ene click chemistry under UV light. We aim to improve the ozone-resistance of NR by PMVS coating and the interphase adhesion between PMVS coating and NR.

\section{Experimental}

\subsection{Materials}

NR was purchased from Shenchi Petrochemical Company (Shandong Province, China). PMVS with vinyl content of $0.18 \mathrm{~mol} \%$ was purchased from Chenguang Research Institute of Chemical Industry (Chengdu, China). Tetrahydrofuran (THF, 99\%), Dicumyl peroxide (DCP, 99\%), 2-methyl-4-(methylthio)-2-morpholinopropiophenone (MMMP, 98\%) as the reaction initiator and trimethylolpropane tris (3-mercaptopropionate) (TMPMP, 95\%) as the crosslinking agent were obtained from $\mathrm{J} \& \mathrm{~K}$ Chemical Technology (Shanghai, China). The chemical structure of PMVS, DCP, MMMP and TMPMP is shown in Figure 1a-1c, and 1d, respectively.

\subsection{Preparation of the samples}

NR sheet with the thickness of $2 \mathrm{~mm}$ was prepared by melt blending of $100 \mathrm{~g}$ of NR and $0.8 \mathrm{~g}$ of DCP using open mill followed by compression molding at $150^{\circ} \mathrm{C}$ for 10 minutes using curing press. The coating of PMVS on NR sheet was as follows. First, $1 \mathrm{~g}$ of PMVS, $0.05 \mathrm{~g}$ of MMMP and $0.05 \mathrm{~g}$ of TMPMP were dissolved in $9 \mathrm{~g}$ of THF under stirring. The concentration of PMVS used is the optimized concentration $(10 \mathrm{wt} \%)$. The as prepared PMVS solution was then coated on the surface of NR sheet by spraying. After the evaporation of THF, the PMVS coating was crosslinked under UV light $(500 \mathrm{~W}$, $320 \mathrm{~nm}<\lambda<420 \mathrm{~nm}$ ) for 15 minutes under nitrogen atmosphere. The distance between the lamp and the samples is $20 \mathrm{~cm}$. We washed the crosslinked samples for several times by using THF to completely remove the unreacted TMPMP and MMMP, and then dried for 8 hours under vacuum.

\subsection{Characterizations}

The ester group and the thiol group were characterized by Fourier Transform Infrared Spectroscopy (Tensor 27, Bruker Optik GmBH, Germany) at a resolution of $1-0.4 \mathrm{~cm}^{-1}$. The fractured surface of PMVS coated NR was studied using a S4700 scanning electron microscopy (SEM) (Hitachi Co. Japan) and energy dispersive X-ray spectroscopy (EDS). The sample of PMVS coated NR was first fractured in liquid nitrogen, and then the fractured surface was coated with a thin layer of gold and observed by SEM and EDS. The adhesion strength was studied by using a cross-cut tape test following ASTM D3359 standard. Ozone resistance of the samples was studied using an ozone test chamber (OZ0500AH, Taiwan High Speed Rail, China) according to ISO 1431-1: 2004 standard. The samples were aged at the ozone concentration of 50 parts per hundred million [pphm] and $200 \mathrm{pphm}$ in static stretching mode, and $50 \mathrm{pphm}$ in dynamic stretching mode. In dynamic stretching mode, the samples were first elongated up to $20 \%$ of the length and then returned to their original length repeatedly with dynamic stretching frequency $0.5 \mathrm{~Hz}$.

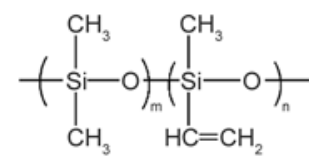

a)

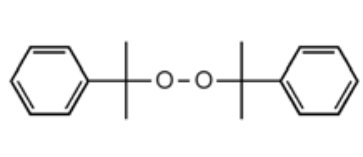

b)

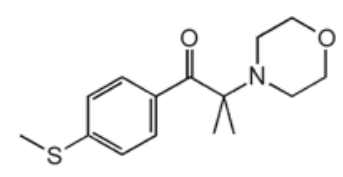

c)

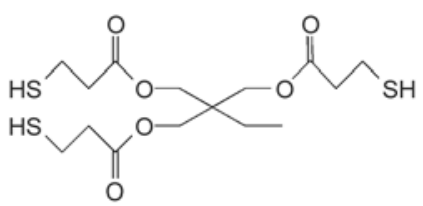

d)

Figure 1. Chemical structure of PMVS (a), DCP (b), MMMP (c) and TMPMP (d) 


\section{Results and discussion}

The reaction between TMPMP and PMVS was characterized by using FTIR, and the results are shown in Figure 2a. The peak at $1727 \mathrm{~cm}^{-1}$ represents the carbonyl group $(\mathrm{C}=\mathrm{O})$ of MMMP, which is a kind of photoinitiator and does not participate in the crosslinking reaction of polymer. Two typical characteristic peaks are observed for TMPMP. The peak at $2570 \mathrm{~cm}^{-1}$ represents the thiol group (-SH), whereas the peak at $1727 \mathrm{~cm}^{-1}$ represents the carbonyl group $(\mathrm{C}=\mathrm{O})$ of TMPMP. Comparing with the spectra of uncrosslinked PMVS (pure PMVS), the characteristic peak of $\mathrm{C}=\mathrm{O}$ group appears on the spectra of crosslinked PMVS, indicating the successful reaction between TMPMP and PMVS by UV irradiation. Here, we should note that the crosslinked PMVS was washed by THF to remove the unreacted TMPMP and MMMP. The disappearance of the characteristic peak of thiol group further demonstrates the reaction between TMPMP and PMVS. Thus, FTIR results demonstrate that we can successfully crosslink PMVS by TMPMP via click chemistry under UV light. The direct evidence of the crosslink of PMVS by TMPMP is observed from Figure $2 b$. We can observe that crosslinked PMVS after UV irradiation is insoluble in THF.

SEM image of the fractured surface of PMVS coated NR is shown in Figure 3a. We can observe that a layer of PMVS with the thickness of 20-30 $\mu \mathrm{m}$ is coated on the surface of NR, and the interphase adhesion between PMVS and NR is good.

The mechanism for the crosslinking of PMVS by TMPMP and the good interphase adhesion between PVMS and NR was studied by using EDS. We selected three samples from PMVS layer (Point A), interphase (Point B) and NR layer (Point C) of the fractured surface of PMVS coated NR, as shown in Figure 3a. Then, we sequentially scanned these samples using EDS to study the content of sulfur element in these samples, and the results are shown in Figure $3 b$. The sulfur element is from TMPMP, and thus there is no sulfur element in both pure NR crosslinked by DCP and pure PMVS without crosslinking, as demonstrated by EDS results shown in Figure 3b. Before UV irradiation, there are some sulfur element in all the samples of PMVS coated NR because of the addition of TMPMP. The content of sulfur element is the highest at the interphase and the lowest at PMVS layer, indicating that TMPMP molecules migrate into NR layer. This is ascribed to the better thermodynamic compatibility between TMPMP and NR than that between TMPMP and PMVS. Interestingly, the content of sulfur element at PMVS layer is increased from $0.16 \%$ to $0.71 \%$ after UV irradiation, whereas that at the interphase is decreased from $0.58 \%$ to $0.1 \%$, suggesting that TMPMP molecules migrate from interphase into PMVS layer during UV irradiation. This indicates the dynamic driving force of crosslinking reaction between thiol groups of TMPMP and double bonds of PMVS, as schematically shown in Figure 4. Meanwhile, the content of sulfur element at NR layer is even slightly increased to $0.34 \%$ after UV irradiation, suggesting the reaction between TMPMP molecules and some double bonds of NR. The lower content of sulfur element at NR layer than that at PMVS layer is ascribed that NR was crosslinked by DCP whereas PMVS was uncrosslinked, indicating the faster crosslink-
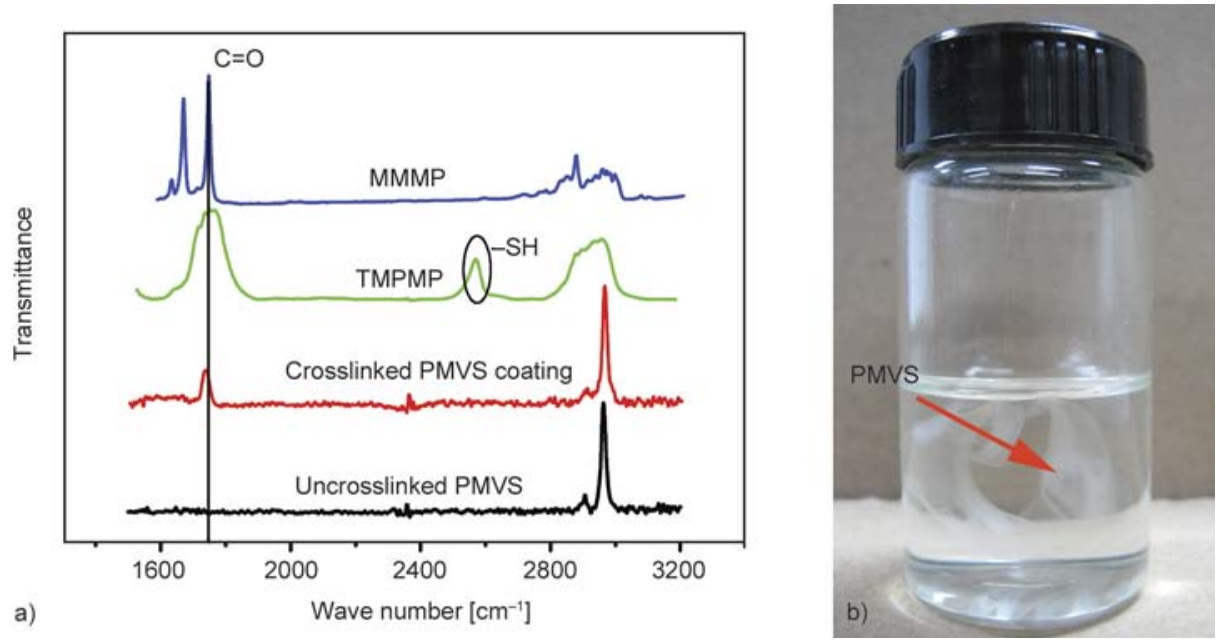

Figure 2. (a) FTIR spectra of uncrosslinked PMVS, TMPMP, MMMP and crosslinked PMVS coating; (b) the photograph of PMVS coating crosslinked by UV irradiation 


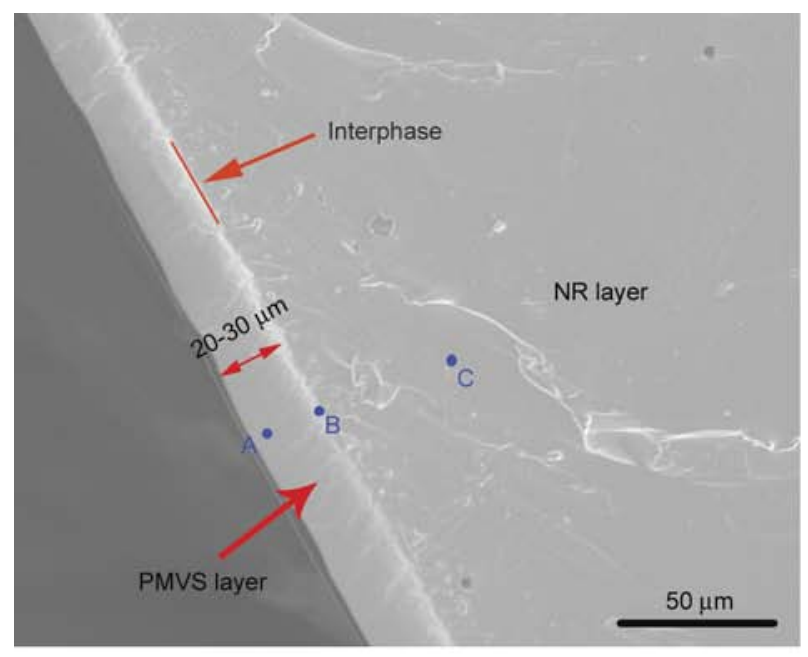

a)

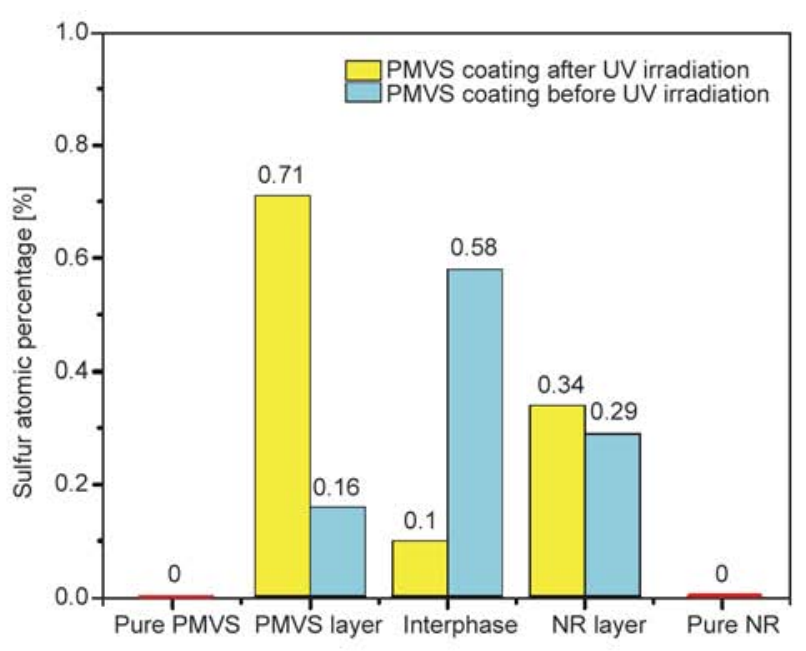

b)

Figure 3. (a) SEM image of fractured surface of PMVS coated NR; (b) sulfur percentage of pure PMVS, pure NR and different layers of PMVS coated NR

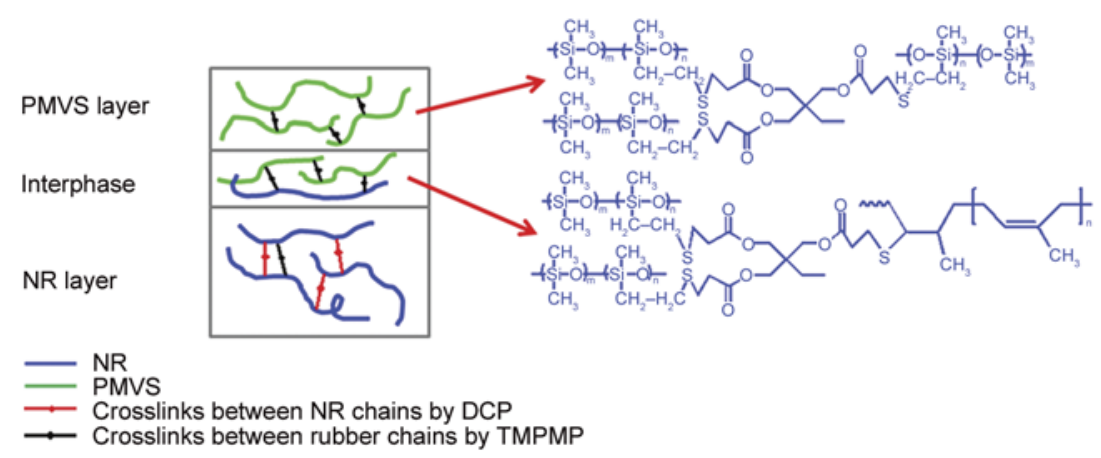

Figure 4. Schematic representation of the crosslinking of PMVS layer and the interface between PMVS coating and NR

ing of PMVS layer than that of NR layer. More importantly, some TMPMP molecules $(0.1 \%)$ remained at the interphase after UV irradiation, which can simultaneously react with both of PMVS and NR, as demonstrated by the improved interphase adhesion strength between NR and PMVS (see below).

The adhesion strength of the PMVS coating on NR was characterized using cross-cut tape test, and the results are shown in Table 1. According to ASTM standard, 5B (100\% adhesion) represents the best adhesion strength, whereas $0 \mathrm{~B}$ represents no adhesion ( $0 \%$ adhesion). The grading of the adhesion of PMVS coating before UV irradiation is $0 \mathrm{~B}$, indicating that the sample does not have any adhesion. However, the grading of adhesion of PMVS coating after UV irradiation reaches $4 \mathrm{~B}$, indicating the good interphase adhesion between PMVS coating after UV irradiation and NR. The direct evidence of the poor adhesion between PMVS coating on NR before UV irradiation, and the good interphase adhesion between PMVS coating on NR after UV irradiation is shown
Table 1. The grading of the adhesion strength of PMVS coating

\begin{tabular}{|l|c|}
\hline & Grading \\
\hline PMVS coating on NR before UV irradiation & $0 \mathrm{~B}$ \\
\hline PMVS coating on NR after UV irradiation & 4B \\
\hline
\end{tabular}

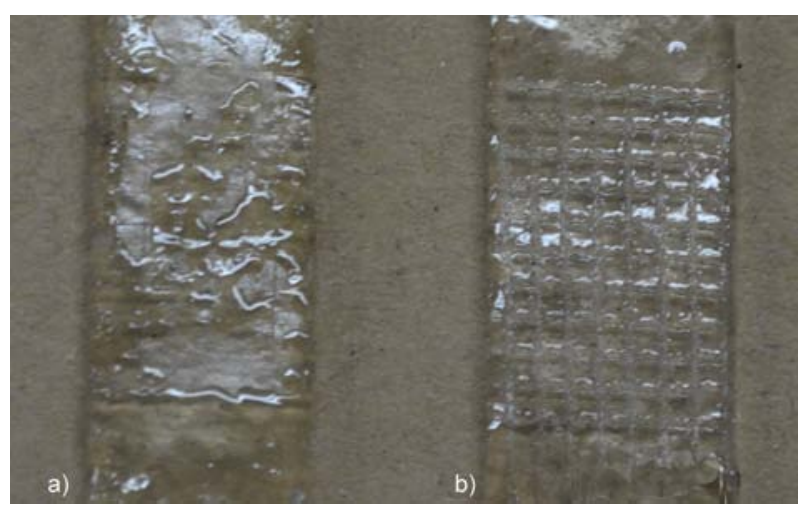

Figure 5. The photos of the surfaces of PMVS coated samples before UV irradiation (a) and after UV irradiation (b)

in Figure 5. Severe detachment of PMVS coating before UV irradiation from NR is observed (see Figure 5a), whereas almost no detachment of PMVS 
coating after UV irradiation from NR is observed (see Figure 5b). The reason is that TMPMP at the interphase can simultaneously react with double bonds of both PMVS and NR under UV irradiation. The ozone resistance of pure NR and PMVS coated NR was evaluated by the SEM images of these samples aged under different ozone aging conditions, as shown in Figure 6. The ozone resistance is shown in Table 2. We observe obvious cracks (B-3) in SEM image of pure NR aged at the ozone concentration of $50 \mathrm{pphm}$ in static stretching mode, whereas we observe no cracks in PMVS coated NR aged at the same conditions, as shown in Figure 6a and Figure $6 \mathrm{~d}$, respectively. We observe wider cracks (B-3) on pure NR aged at the ozone concentration of $200 \mathrm{pphm}$ in static stretching mode, whereas we observe only some micro-cracks (A-1) on PMVS coated NR aged at the same conditions, as shown in Figure $6 \mathrm{~b}$ and Figure 6e, respectively. The cracks on the surface of pure NR aged at the ozone concentration of $50 \mathrm{pphm}$ in dynamic stretching mode even become much wider (B-3), indicating the very poor ozone aging resistance of pure NR, as shown in Figure $6 \mathrm{c}$ and Table 2. More cracks (B-1) on PMVS coated NR aged at the same condition was observed, but they are still micro-cracks, as shown in Figure $6 \mathrm{f}$. These results demonstrate that the coating of PMVS on NR by crosslinking via click chemistry can largely improve the ozone aging resistance of NR,
Table 2. The ozone resistance of the samples

\begin{tabular}{|l|l|c|}
\hline & \multicolumn{1}{|c|}{ Testing conditions } & $\begin{array}{c}\text { Type of } \\
\text { cracking }\end{array}$ \\
\hline \multirow{3}{*}{ Pure NR } & $50 \mathrm{pphm}$ in static stretching mode & $\mathrm{B}-3$ \\
\cline { 2 - 3 } & $200 \mathrm{pphm}$ in static stretching mode & $\mathrm{B}-3$ \\
\cline { 2 - 3 } & $50 \mathrm{pphm}$ in dynamic stretching mode & $\mathrm{B}-3$ \\
\hline \multirow{2}{*}{$\begin{array}{l}\text { NR coated } \\
\text { with PMVS }\end{array}$} & $50 \mathrm{pphm}$ in static stretching mode & $\mathrm{nc}^{\mathrm{a}}$ \\
\cline { 2 - 3 } & $200 \mathrm{pphm}$ in static stretching mode & $\mathrm{A}-1$ \\
\cline { 2 - 3 } & $50 \mathrm{pphm}$ in dynamic stretching mode & $\mathrm{B}-1$ \\
\hline
\end{tabular}

aThe cracking was not appeared on the surface of rubber specimens.

Marking the types of cracking:

A: A small number of cracking.

B: A large of number cracking.

C: Numberless cracking.

1. That which cannot be seen with eyes but can be confirmed with 10 times magnifying glass.

2. That which can be confirmed with naked eyes.

3. That which the deep and comparatively long (below $1 \mathrm{~mm}$ ).

4. That which the deep and long (above $1 \mathrm{~mm}$ and below $3 \mathrm{~mm}$ ).

5. That which about to crack more than $3 \mathrm{~mm}$ or about to severe.

facilitating the storage and application of NR. The poor ozone resistance of NR is because of its unsaturated chain structure of the polyisoprene backbone, which is easily attacked by oxygen or ozone. PMVS, with very low content of unsaturated bond, has a basic building block of silicon-oxygen bond with high bond energy, which is not easily attacked by oxygen or ozone, and thus shows a good ozone resistance. Therefore, the PMVS coating on NR can effectively protect NR from ozone attack. Com-

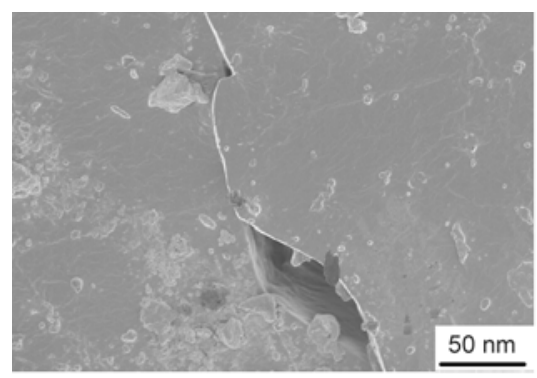

a)

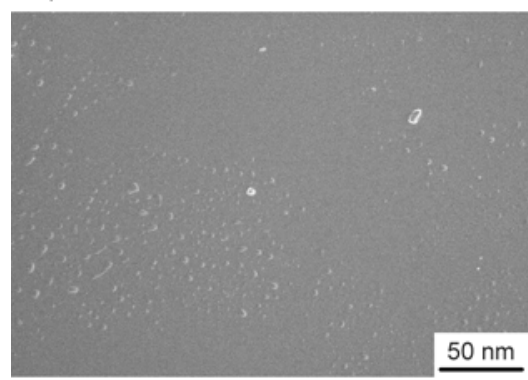

d)

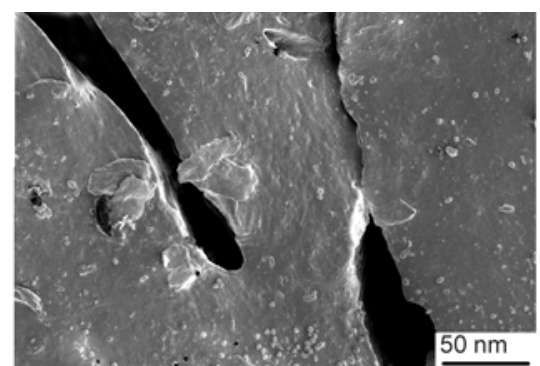

b)

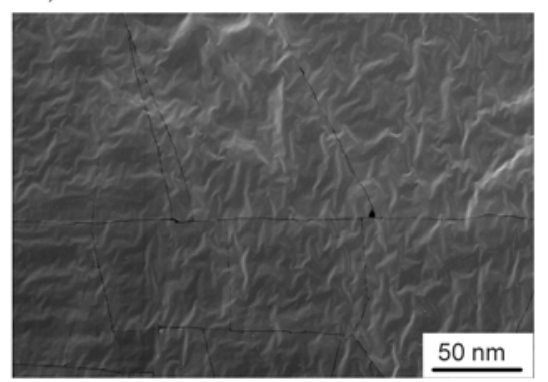

e)

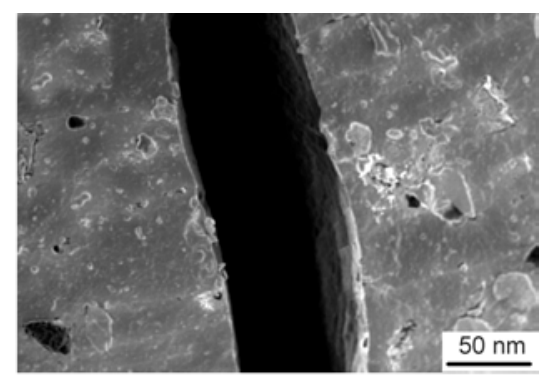

c)

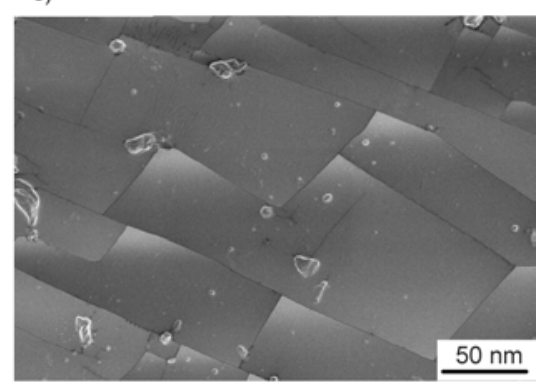

f)

Figure 6. SEM images of pure NR (a, b, c) and PMVS coated NR (d, e, f) under different ozone aging conditions. (a) (d) at the ozone concentration of $50 \mathrm{pphm}$ in static stretching mode; (b) (e) at the ozone concentration of $200 \mathrm{pphm}$ in static stretching mode; (c) (f) at the ozone concentration of $50 \mathrm{pphm}$ in dynamic stretching mode. 
pared with the previous studies using the traditional methods to improve the ozone resistance [1, 15], our method also can achieve the good ozone resistance. The advantage of our method is the achievement of good interphase adhesion between PMVS coating and NR. Thus, the mechanical properties of NR are not affected by the PMVS coating in this study.

\section{Conclusions}

We have successfully prepared PMVS coating on NR via thiol-ene click reaction under UV irradiation, which simultaniously realized the crosslinking of PMVS layer and the covalent crosslinking between PMVS layer and NR layer. As a result, the ozone resistance of NR was improved by the coating of crosslinked PMVS layer on the surface of NR sheet. Meanwhile, we obtained a good interphase adhesion between PMVS coating and NR. Our study provides an effective technique to prepare polymer materials with good ozone-resistance.

\section{Acknowledgements}

We gratefully acknowledge the financial supports of the National Natural Science Foundation of China under Grant No. 51373012 and 51221002.

\section{References}

[1] Piya-areetham P., Rempel G. L., Prasassarakich P.: Hydrogenated nanosized polyisoprene as a thermal and ozone stabilizer for natural rubber blends. Polymer Degradation and Stability, 102, 112-121 (2014). DOI: 10.1016/j.polymdegradstab.2014.01.032

[2] Saeed F., Ansarifar A., Ellis R. J., Haile-Meskel Y., Farid A. S.: Effect of the blooming of chemical curatives on the cyclic fatigue life of natural rubber filled with a silanized silica nanofiller. Journal of Applied Polymer Science, 120, 2497-2507 (2011).

DOI: 10.1002/app.33396

[3] Abdelwahab N. A., El-Nashar D. E., Abd El-Ghaffar M. A.: Polyfuran, polythiophene and their blend as novel antioxidants for styrene- butadiene rubber vulcanizates. Materials and Design, 32, 238-245 (2011). DOI: $10.1016 /$ j.matdes.2010.06.003

[4] Ramesan M. T., Alex R., Khanh N. V.: Studies on the cure and mechanical properties of blends of natural rubber with dichlorocarbene modified styrene-butadiene rubber and chloroprene rubber. Reactive and Functional Polymers, 62, 41-50 (2005).

DOI: $10.1016 /$ j.reactfunctpolym.2004.08.002
[5] Hinchiran N., Lertweerasirikun W., Poonsawad W., Rempel G. L., Prasassarakich P.: Hydrogenated natural rubber blends: Aspect on thermal stability and oxidative behavior. Journal of Applied Polymer Science, 113, 1566-1575 (2009).

DOI: $10.1002 /$ app.30034

[6] Joseph R.: HXNBR coating for improving aging resistance of natural rubber products. Rubber Chemistry and Technology, 79, 553-560 (2006).

DOI: $10.5254 / 1.3547951$

[7] Momen G., Farzaneh M., Jafari R.: Wettability behaviour of RTV silicone rubber coated on nanostructured aluminium surface. Applied Surface Science, 257, 6489-6493 (2011).

DOI: $10.1016 /$ j.apsusc.2011.02.049

[8] Kim E. S., Kim E. J., Lee T. H., Yoon J. S.: Improvement of the adhesion properties of silicone rubber by the incorporation of silane-modified montmorillonite. Journal of Applied Polymer Science, 128, 2563-2570 (2013). DOI: $10.1002 / a p p .38489$

[9] Lowe A. B.: Thiol-ene 'click' reactions and recent applications in polymer and materials synthesis. Polymer Chemistry, 1, 17-36 (2010).

DOI: $\underline{10.1039 / \mathrm{b} 9 \mathrm{py} 00216 \mathrm{~b}}$

[10] Li G. L., Xu L. Q., Tang X., Neoh K. G., Kang E. T.: Hairy hollow microspheres of fluorescent shell and temperature-responsive brushes via combined distillationprecipitation polymerization and thiol-ene click chemistry. Macromolecules, 43, 5797-5803 (2010).

DOI: $10.1021 / \mathrm{ma} 1008012$

[11] Li G. L., Wan D., Neoh K. G., Kang E. T.: Binary polymer brushes on silica@polymer hybrid nanospheres and hollow polymer nanospheres by combined alkyneazide and thiol-ene surface click reactions. Macromolecules, 43, 10275-10282 (2010).

DOI: $10.1021 / \mathrm{ma} 1020787$

[12] Xue L., Zhang Y., Zuo Y., Diao S., Zhang J., Feng S.: Preparation and characterization of novel UV-curing silicone rubber via thiol-ene reaction. Materials Letters, 106, 425-427 (2013).

DOI: $10.1016 /$ j.matlet.2013.05.084

[13] Campos L. M., Meinel I., Guino R. G., Schierhorn M., Gupta N., Stucky G. D., Hawker C. J.: Highly versatile and robust materials for soft imprint lithography based on thiol-ene click chemistry. Advanced Materials, 20, 3728-3733 (2008).

DOI: $10.1002 / \mathrm{adma} .200800330$

[14] Zhang J., Chen Y., Brook M. A.: Facile functionalization of PDMS elastomer surfaces using thiol-ene click chemistry. Langmuir, 29, 12432-12442 (2013). DOI: $10.1021 / 1 \mathrm{a} 403425 \mathrm{~d}$

[15] Simma K., Rempel G. L., Prasassarakich P.: Improving thermal and ozone stability of skim natural rubber by diimide reduction. Polymer Degradation and Stability, 94, 1914-1923 (2009).

DOI: 10.1016/j.polymdegradstab.2009.08.005 